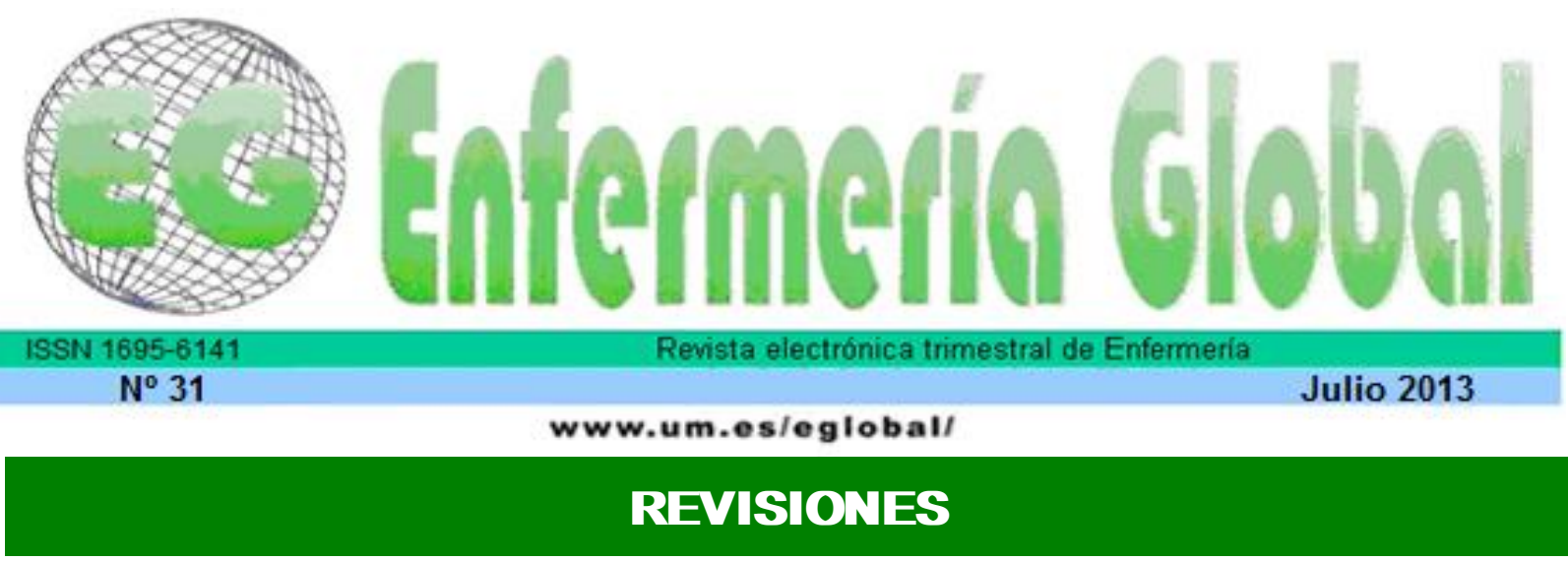

\title{
Experiencias y sentimientos vividos durante una gestación de alto riesgo: un estudio documental 2005-2011
}

Experiences and feelings endured during a high-risk pregnancy: a documental study 20052011

\section{*Laza Vásquez, Celmira **Quintero Sánchez, Jimena **Jiménez Caicedo, Hasbleidy Johana **Preciado Parrado, Javier Alexander}

*Magíster en Enfermería con énfasis en cuidado de la salud materno perinatal. Profesora asociada Docente área investigativa. E mail: claza1@fucsalud.edu.co ** Estudiante Facultad de Enfermería. Miembro del Semillero de Investigación "Red del Cuidado Ciencia y Arte de Enfermería"-RCUAE. Fundación Universitaria de Ciencias de la Salud. Bogotá. Colombia.

Palabras clave: Embarazo de alto riesgo; experiencias; vivencias; estudio documental; investigación cualitativa

.Keywords: High-risk pregnancy; experience; documental study; qualitative research

\section{RESUMEN}

Objetivo: Acercase a las experiencias vividas por las mujeres frente a una gestación de alto riesgo, reportadas en la literatura científica durante el período de 2005 a 2011.

Materiales y métodos: Estudio documental de corte cualitativo. Las unidades de análisis fueron los artículos científicos de todas las nacionalidades en idioma español, inglés y portugués en el período 2005-2011. Los datos se trataron mediante el análisis de contenido.

Resultados: La búsqueda bibliográfica dio como resultado veinte artículos que respondían al objetivo del estudio. Todos tenían un enfoque cualitativo, 14 de ellos fueron trabajos realizados en el mundo en desarrollo y el año de mayor publicación fue 2010. Del análisis emergieron dos grandes temas: las experiencias de las mujeres frente a un embarazo de alto riesgo producto de una condición patológica y las que fueron producto de una gestación en una edad reproductiva temprana o tardía.

Conclusiones: Se resalta el papel que juegan las redes de apoyo para las gestantes: la familia, otras mujeres en igual condición y los profesionales de la salud; las dificultades durante la hospitalización y el manejo de un lenguaje cargado de tecnicismos por parte de los profesionales. El cuidado de enfermería debe encaminarse a reforzar las redes de apoyo y procurar mejores ambientes de hospitalización. 
Las experiencias de las gestantes conviviendo con $\mathrm{VIH}$ y de las mujeres que tienen un embarazo después de los 35 años, son necesidades aún pendientes por la investigación. El enfoque cualitativo es válido para el abordaje de este tema.

\section{ABSTRACT}

Objective: To explore the experiences endured by women while in a high-risk pregnancy, reported in the scientific literature during 2005-2011.

Methods: Qualitative documental study. Analysis units were the scientific papers published worldwide in Spanish, English and Portuguese during 2005 to 2011. Data were treated by contents analysis.

Results: Bibliographic search rendered twenty papers dealing with the study objective. All of them had qualitative focus, fourteen of them were papers published in the so-called underdeveloped world; most frequent publication occurred around 2010. From the analysis emerged two main topics: the women's experiences while in a high-risk pregnancy from a pathologic condition, and those product of a pregnancy in early or late reproductive age.

Conclusion: The role of the pregnant support networks is underlined: family, other women sharing their condition and health professionals; also were of note the difficulties during hospitalization and the technical jargon used by those professionals. Nursing care should focus to reinforce the networks and to procure better environment for the inpatients. The experiences endured by pregnant women living with HIV or by those women older than 35-year are still matter of pending research based upon qualitative focus.

\section{INTRODUCCIÓN}

En Colombia, la mortalidad materna es la tercera causa de muerte en la mujer adulta joven, siendo la razón de mortalidad materna en 2008 de 71,6 por 100.000 nacidos vivos $^{1}$ mostrando solo un muy leve descenso en comparación al año anterior. A pesar de que la muerte materna es relevante, también lo es la morbilidad durante la gestación, que incluye distintos grados de gravedad e indica la necesidad de conocer mejor los problemas relacionados con el embarazo, parto y puerperio que no resultan en muerte; pero que pueden causar sufrimiento y dejar secuelas en las mujeres ${ }^{2}$. La morbilidad materna extrema o materna severa causada por una gestación de alto riesgo se ha definido como una complicación obstétrica severa y que requiere una intervención médica urgente, encaminada a prevenir la muerte materna ${ }^{3}$.

La gestación de alto riesgo es considerada aquella en la que madre, el feto $\mathrm{y} / \mathrm{o}$ el neonato tienen una mayor probabilidad de enfermar, morir o padecer secuelas antes o después del parto ${ }^{4}$. El riesgo de una gestación se eleva cuando coexisten una condición patológica, un intervalo intergenésico corto, una alta paridad o una edad reproductiva temprana o tardía 5 .

Cordero y González ${ }^{6}$ Ilaman la atención acerca de la gestación a edad muy temprana y después de los 35 años de edad ya que supone un incremento del riesgo adicional del parto y para la mortalidad materna; además es especialmente importante en los países en desarrollo, donde las mujeres mayores tienen con frecuencia numerosos hijos. Este es uno de los factores usados para definir el alto riesgo materno. Otros factores predictores de morbilidad han sido la raza no blanca, exclusión social, historia previa de hemorragia posparto, embarazo múltiple, hipertensión, diabetes mellitus, cesárea de emergencia y anemia ${ }^{7}$.

Por otro lado, el embarazo en la adolescencia es una condición de alto riesgo debido a que esta es una etapa del crecimiento y desarrollo humano con características muy 
especiales por los cambios fisiológicos, psicológicos y sociales que abarcan a esta población. Así, el embarazo en esta etapa de la vida es una condición de riesgo por las posibles complicaciones que pueden ocurrir desde el punto de vista médico, obstétrico y perinatal; lo que tiene mayor significado mientras más temprana sea la edad de la adolescente ${ }^{8}$.

Además del abordaje de los aspectos médicos de los embarazos de alto riesgo, un elemento importante son las experiencias de las mujeres que han vivido estos eventos. La experiencia, según Husserl ${ }^{9}$ es una conducta activa y valorativa más que una cognoscitiva y juzgadora; y sobre ella descansa toda praxis humana. En la esfera de la experiencia, el mundo constituye siempre el sustrato que le sirve de fundamento; ya sea aquella que inicia el conocimiento como que dinamiza la praxis. Las experiencias/vivencias, en un sentido estricto tienen un carácter de actualidad, pero en esta actualidad, marcan de alguna manera su presencia vivencias pasadas, estructuralmente unidas a la actual y a las expectativas del futuro ${ }^{10}$.

Así, además de la evaluación y manejo de los problemas de salud que presenta la mujer con una gestación de alto riesgo, es importante, de la misma manera, valorar su vivencia e impacto emocional a futuro para un conocimiento y comprensión de la experiencia frente a esta situación. Souza, Cecatti, Parpinelli, Krupa y Osis ${ }^{11}$ proponen que tener en cuenta las voces de las que han vivido y sobrevivido a una gestación de alto riesgo es necesario ya que pueden brindar un testimonio acerca de qué, cómo, cuándo y por qué los hechos ocurrieron en un cierto modo. La identificación de los trastornos por estrés agudo y trastornos de estrés postraumático pueden jugar un papel importante en el desarrollo de secuelas a largo plazo y contribuir a disminuir la carga global de la morbilidad materna severa.

Sin embargo, frente a esto afirma Vecino ${ }^{12}$ que en Colombia la información disponible sobre lo que piensan y sienten las embarazadas acerca del riesgo de la gestación, el control prenatal, la atención recibida o el parto hospitalario, entre otros, es escasa y tiene muy poca visibilidad o es nula.

Establecer el estado del arte acerca de las vivencias frente a la gestación de alto riesgo se convierte en una necesidad para contar con evidencias y conocimientos obtenidos en otros contextos, dados los escasos estudios realizados en Colombia. En este sentido, la investigación documental se convierte en un instrumento importante ya que sus resultados ofrecen una visión global del estado actual del conocimiento, dando cuenta de un saber acumulado en determinado momento histórico acerca de un área específica del saber. Como tal no se considera un producto terminado, sino que da origen a nuevos campos de investigación y éstos a su vez generan otros en el área sobre la cual se ha investigado ${ }^{13}$.

Asimismo, los resultados permitirán identificar vacíos en el conocimiento y nuevos problemas susceptibles a investigar, y serán insumo fundamental para futuros proyectos y establecer una línea investigativa de base en el tema. Por lo anterior, el presente estudio tuvo como objetivo acercase a las experiencias vividas por las mujeres frente a una gestación de alto riesgo, reportadas en la literatura científica durante el período de 2005 a 2011. 


\section{MATERIALES Y MÉTODOS}

Se realizó un estudio documental de corte cualitativo, tomando como referencia la propuesta de $\operatorname{Hoyos}^{13}$, que este diseñó como una forma de dar cuenta de la investigación que se ha realizado sobre un tema central. Para esto, se tuvieron en cuenta los artículos científicos originales (reporte de resultados de investigaciones) de cualquier nacionalidad publicados en idioma español, inglés y portugués entre los años 2005 a 2011 e indexados en las revistas de Ciencias de la Salud y Ciencias Humanas y Sociales en las siguientes bases de datos: EBSCO, Academic Edition, MedicLatina, Medline, Journal Medline, Dynamic, Pubmed, Ovid, Ovid Nursing, Scielo, Cantarida y Lilacs. ScienceDirect (Elsevier, Pergamon, Excerpta-Medica y North Holland).

Los descriptores DeCs utilizados, en idioma español, inglés y portugués fueron: embarazo, gestación, percepción, alto riesgo, morbilidad materna extrema, experiencias, vivencias, enfermería e investigación cualitativa. Se utilizó la opción de búsqueda avanzada, teniendo en cuenta la restricción del período de tiempo definido, el objetivo del estudio y con el uso del booleano AND.

Para la recolección de los datos, se elaboró una ficha instructiva tomando como referencia la propuesta de Hoyos $^{13}$ que tuvo en cuenta los siguientes aspectos: aspectos formales, asunto investigado, delimitación contextual, propósito, metodología y resultados.

El tratamiento de la información se realizó mediante el análisis de contenido, el cual hace referencia a un conjunto de métodos y procedimientos de análisis de documentos que ponen énfasis en el sentido del texto ${ }^{14}$. El proceso de análisis se realizó simultáneamente con la recolección de los datos y para esto se creó una matriz en la cual se registró la información de cada unidad de análisis. Lo anterior se constituyó en la reducción inicial de los datos.

Posteriormente, se llevó a cabo una reducción y síntesis de la información que permitió perfilar las categorías iníciales, para concluir con la agrupación en dos grandes temas y sus categorías.

\section{ANÁLISIS DE LOS RESULTADOS}

\section{- Aspectos formales de los artículos}

La búsqueda bibliométrica dio como resultado inicial 150 documentos, de los cuales después de la lectura inicial, 20 respondían al objetivo del estudio. Dentro de los aspectos formales de las unidades de análisis, se resalta que 9 de ellos fueron publicados en idioma portugués, seis en inglés y el resto en español. Todos los estudios fueron realizados con un enfoque cualitativo de la investigación, predominando dentro de las tradiciones, la Fenomenología y la Teoría fundamentada (tres de cada uno). Sin embargo, 9 de los trabajo no especifican ni diseño ni tradición investigativa. 
Tabla 1. Distribución de las publicaciones consultadas según la tradición y/o diseño investigativo bajo la cual se realizó el estudio. Bogotá, 2012.

\begin{tabular}{|l|l|}
\hline Tipo de estudio & No. \\
\hline $\begin{array}{l}\text { No especifica tradición o } \\
\text { diseño }\end{array}$ & 9 \\
\hline Teoría Fundamentada & 3 \\
\hline Fenomenológico & 3 \\
\hline Descriptivo & 2 \\
\hline Hermenéutico-interpretativo & 2 \\
\hline Historia oral & 1 \\
\hline Total & $\mathbf{2 0}$ \\
\hline
\end{tabular}

Fuente: Los autores, 2012.

El mayor número, catorce de ellos, de estudio fueron realizados en países en desarrollo, lo que es coincidente con el peso de la mortalidad y morbilidad materna; y la presentación de gestaciones de alto riesgo en estos contextos.

Tabla 2. Distribución de las publicaciones consultadas según el país donde se desarrollaron. Bogotá, 2012.

\begin{tabular}{|l|l|}
\hline Lugar del estudio & No. \\
\hline Brasil & 9 \\
\hline Colombia & 3 \\
\hline Suecia & 2 \\
\hline México & 1 \\
\hline Australia & 1 \\
\hline Reino Unido & 1 \\
\hline Tailandia & 1 \\
\hline Taiwán & 1 \\
\hline Israel & 1 \\
\hline Total & 20 \\
\hline
\end{tabular}

Fuente: Los autores, 2012.

El año de mayor publicación de artículos el 2010, lo que demuestra un aumento en el interés en la investigación sobre el tema, a pesar de lo novedoso del mismo. No se encontraron publicaciones del año 2011.

Tabla 3. Distribución de las publicaciones consultadas según el año de publicación. Bogotá, 2012.

\begin{tabular}{|l|l|}
\hline $\begin{array}{l}\text { Año } \\
\text { publicación }\end{array}$ & No. \\
\hline 2010 & 6 \\
\hline 2009 & 5 \\
\hline 2008 & 3 \\
\hline 2007 & 2 \\
\hline 2006 & 2 \\
\hline 2005 & 2 \\
\hline Total & 20 \\
\hline
\end{tabular}

Fuente: Los autores, 2012. 
Es interesante que 17 de los trabajos fueron realizados por profesionales de Enfermería.

\section{Temas y categorías resultantes}

Los resultados se agruparon en dos grandes temas: las experiencias de las mujeres frente a un embarazo de alto riesgo producto de una condición patológica o alteración de salud y las que fueron producto de una gestación en una edad reproductiva temprana o tardía.

Tabla 4. Distribución de las publicaciones consultadas según la agrupación de los resultados y temas abordados en las publicaciones, Bogotá, 2012.

\begin{tabular}{|c|c|c|}
\hline Temas resultantes & $\begin{array}{l}\text { Causas del alto riesgo en la gestación } \\
\text { abordadas en los estudios. }\end{array}$ & No. \\
\hline \multirow{6}{*}{$\begin{array}{l}\text { Experiencias de las mujeres } \\
\text { frente a un embarazo de } \\
\text { alto riesgo producto de una } \\
\text { condición patológica. }\end{array}$} & Varias condiciones patológicas abordadas. & 4 \\
\hline & Síndrome hipertensivo de la gestación. & 3 \\
\hline & $\begin{array}{l}\text { Diabetes mellitus gestacional y Diabetes } \\
\text { mellitus. }\end{array}$ & 2 \\
\hline & $\begin{array}{l}\text { Virus de } \\
\text { adquirida/Síndrome de }\end{array} \begin{array}{l}\text { Inmunodeficiencia } \\
\text { adquirida. }\end{array}$ & 2 \\
\hline & Ruptura prematura de membranas & 1 \\
\hline & Embarazo multifetal & 2 \\
\hline \multirow[b]{2}{*}{$\begin{array}{l}\text { Experiencias de las mujeres } \\
\text { frente a un embarazo de } \\
\text { alto riesgo producto de la } \\
\text { edad reproductiva temprana } \\
\text { o tardía. }\end{array}$} & Embarazo adolescente & 5 \\
\hline & Embarazo a edad tardía & 1 \\
\hline \multicolumn{2}{|l|}{ Total } & 20 \\
\hline
\end{tabular}

Fuente: Los autores, 2012.

En el primer grupo, se observó una diversidad de temas abordados, aunque hay algunos que todavía se consideran pendientes en la producción científica; y llama la atención el número de estudios realizados acerca de las experiencias vividas durante una gestación con preeclampsia, visible también en los artículos que abordaban varias condiciones patológicas de las gestantes del estudio.

El interés por las vivencias de las gestantes que han padecido esta alteración se debe a que en 2009, más de medio millón de mujeres morían en el embarazo, parto y puerperio a causa de la presentación de eventos hipertensivos de la gestación y por sus complicaciones; y el $99 \%$ de estas muertes ocurrió en países en vías de desarrollo ${ }^{15}$. Los estados hipertensivos de la gestación persisten como la principal causa de muerte materna en los países pobres, siendo responsables de 63.000 muertes anuales y la mortalidad materna por preeclampsia es entre el $0 \%$ y $13.2 \%{ }^{16}$. La presentación de esta última alteración es entre el $5 \%$ y $8 \%$ de las gestaciones y puede llevar a resultados perinatales adversos como bajo peso al nacer, prematuridad, muerte fetal y retardo del crecimiento intrauterino ${ }^{17}$. También es causa de secuelas permanentes en la mujer, como alteraciones neurológicas, hepáticas, renales o hematológicas ${ }^{18}$. 
En el segundo tema, la emergencia de las diferentes vivencias del embarazo en adolescente no es más que la evidencia de la realidad de este fenómeno en el mundo en desarrollo y respuesta de la investigación a esta situación. Frente al incremento sostenido de este fenómeno, la Cepal ${ }^{19}$, explica que este se da en países con distinto nivel de desarrollo económico, nivel educativo e incidencia de la pobreza; lo que revela su complejidad y multiplicidad de factores que inciden en su evolución. La mortalidad materna y el embarazo adolescente son más elevados entre las mujeres de sectores más pobres, quienes tienen menor acceso a la educación, a la información y a la salud sexual y reproductiva.

A continuación se describen los temas y las categorías que dan cuentan de las experiencias vividas por las mujeres que han padecido una gestación de alto riesgo.

\section{Tema 1. Experiencias de las mujeres frente a un embarazo de alto riesgo producto de una condición patológica}

Este tema agrupa diferentes categorías que se comportan como opuestas y que dan cuentan de experiencias y sentimientos vividos por las gestantes durante su transcurrir por el alto riesgo. Sin embargo, estas categorías no solo dan cuenta de las vivencias de las mujeres sino también de las familias, y las relaciones que se establecen con los profesionales de salud y con otras mujeres en iguales situaciones.

\section{Percepción de la enfermedad y su causalidad}

Solo en los tres trabajos que abordaron las experiencias de las gestantes que vivenciaron una preeclampsia, emergieron aspectos de la percepción y causalidad de la enfermedad. Así, las condiciones patológicas causantes de un embarazo de alto riesgo fueron percibidas como preocupantes, de gravedad, amenazantes para su vida y la de su hijo; en especial, un riesgo de muerte para su hijo. Sumándose a lo anterior, que la enfermedad tuvo una aparición súbita e inesperada.

A pesar que en algunos casos las mujeres identificaron los signos de alarma, desconocían su significado e importancia.

La causalidad del alto riesgo en el embarazo no fue clara y fue atribuido al estrés y los problemas en el hogar y con la pareja. La herencia, los factores emocionales, y psicológicos y la falta de buena alimentación durante la gestación fueron otros. El castigo divino fue un factor de gran peso en la percepción etiológica de la enfermedad.

\section{Experiencias negativas vividas durante la enfermedad}

Esta categoría se considera, junto con la de sentimientos negativos generados por la enfermedad, la de mayor peso e importancia en los estudios. A continuación se describen los elementos que la integran.

La hospitalización. En ocho de los estudios, las gestantes refirieron que la experiencia de estar hospitalizadas fue perturbadora, causando ira, frustración y soledad por el escaso tiempo de los horarios de las visitas y la poca actividad física que se les permitía realizar. 
Souza, Araújo, Azevedo, Jerônimo, Barbosa y Sousa ${ }^{20}$, referenciaron en su investigación que algunas mujeres no aceptaban la hospitalización porque no presentaban ningún signo o síntoma, mientras que para otras, ver a sus hijos en la UCIN significó tristeza y desesperación.

Soporte deficiente de redes de apoyo primarias. Cuatro de los trabajos refirieron que al presentar una alteración en el embarazo, las familias se vieron frente a una situación estresante y desgastante, lo que ocasionó profundas alteraciones en la dinámica familiar y que se prolongó con la internación del hijo.

En el caso de Diabetes mellitus gestacional (DMG), Persson, Winkvist y Mogren ${ }^{21}$ documentaron que las mujeres se sentían constantemente controladas por la dieta para mantener niveles adecuados de glucosa en sangre. Por su parte, en relación a las gestantes conviviendo con el Virus de Inmunodeficiencia Adquirida (VIH), Araujo, Silveira, Silveira y Melo ${ }^{22}$, referenciaron que las mujeres no recibieron ningún apoyo familiar y tuvieron que enfrentar muchos sufrimientos, lo cual les dificultó la interacción social.

Deficiente relación con los profesionales de la salud. En cuatro estudios, las mujeres cuestionaron a los profesionales al expresar la falta de claridad en la información brindada por estos acerca de su estado de salud y la de su hijo, al estar esta información cargada de "tecnicismos" que no comprendían. Lo mismo ocurría durante los procedimientos o intervenciones, lo cual que aumentaba su ansiedad y angustia.

\section{Sentimientos negativos generados por la enfermedad}

En trece de los estudios, las gestantes refirieron miedo, tristeza, preocupación, inseguridad y angustia por las complicaciones que presentaron y las consecuencias para la vida del binomio madre-hijo.

Asimismo, Persson, Winkvist y Mogren ${ }^{21}$ documentaron sentimientos de culpa en las gestantes al ser diagnosticadas con DMG, ya que se consideraron responsables de las causas de su enfermedad. Muy relacionado a la culpa, se encontró la pérdida de control, impotencia, vergüenza y duda; todos sentimientos relacionados con la lucha de las mujeres para resistir las persistentes tentaciones y con los bajos ajustes que hacían a las recomendaciones médicas dadas.

La incertidumbre durante el momento del parto fue alta ya que representaban los resultados maternos y fetales, al esperar que la DMG no hubiera afectado a la salud de su hijo y que se pudiera resolver su enfermedad y no quedar con secuelas.

La culpa también acompañó a las mujeres contagiadas con $\mathrm{VIH}$, evidenciado en el estudio de Ross, Sawatphanit, Draucker y Suwansujarid ${ }^{23}$; pero además encontraron que en las participantes se encontraban presentes sentimientos de vergüenza y enojo en el momento de recibir la noticia del diagnóstico, con su pareja, familia y amigos. La soledad las acompañó durante gran parte del embarazo, al no tener con quien compartir su situación.

Los sentimientos de ambivalencia fueron expresados por las mujeres que tuvieron períodos prolongados de hospitalización, como fue el caso de las gestantes que padecieron DMG y Ruptura prematura de membrana, ya que la hospitalización 
proporcionaba seguridad para su hijo pero a la vez sintieron la necesidad de ocuparse de las tareas del hogar y el cuidado de sus otros hijos.

\section{Experiencias positivas frente a la enfermedad}

Las experiencias positivas de las gestantes durante su padecimiento emergieron en diecisiete de las investigaciones revisadas, siendo muy diversas sus fuentes. En general estas aparecieron cuando hubo una adaptación o resolución de la situación de las mujeres, o cuando se hicieron evidentes las redes de apoyo.

La hospitalización. En el caso de dos estudios acerca de experiencias de gestantes con preeclampsia ${ }^{24-20}$, la hospitalización fue vista por las mujeres como el lugar donde se les proporcionó un apoyo social y emocional por parte del personal de salud y por otras pacientes embarazadas. Además, Ross, Sawatphanit, Draucker y Suwansujarid $^{23}$, registraron en su artículo sobre VIH en el embarazo, que las mujeres participaron en las reuniones y programas antirretrovirales, ayudándoles a sobrellevar la enfermedad.

Redes de apoyo primarias. En siete de los estudios, las gestantes refirieron sentir tranquilidad cuando se encontraron con sus familiares. Estos acompañaron a las mujeres durante su embarazo y las apoyaron en sus decisiones; el apoyo de los padres fue un elemento muy importante en las mujeres, ya que sentían la necesidad de que las escucharan y que les dieran una voz de aliento para así reconfortar su estado de ánimo.

Araujo, Silveira, Silveira y Melo ${ }^{22}$, registraron en su artículo sobre VIH que las gestantes que contaron con el apoyo familiar comprendieron mejor su enfermedad, la causa y su manejo; generando una mejor adherencia al tratamiento.

Otro soporte social fueron las gestantes. Barlow, Hainsworth y Thornton ${ }^{24}$ en su estudio sobre hipertensión durante la gestación documentaron que las mujeres se sintieron esperanzadas al escuchar testimonios de otras que tuvieron preeclampsia y los resultados finales fueron satisfactorios.

Relación satisfactoria con los profesionales de la salud. En cinco de los estudios se mostró que hubo preocupación y esmero por parte de los profesionales para que las gestantes cumplieran con el tratamiento; la consejería fue encaminada principalmente a minimizar los problemas emocionales generados por el diagnóstico. Gracias al apoyo y constancia del personal de salud se disminuyó la angustia y aumentó el estado de ánimo.

Espiritualidad y prácticas religiosas. Aferrarse a Dios generó en las gestantes una experiencia positiva para aliviar la carga que supuso la enfermedad, y así, sentir mejoría de su situación. La fe y las prácticas religiosas se convirtieron en fuente de esperanza y confianza para llevar el embarazo a un feliz término.

\section{Sentimientos positivos generados por la enfermedad}

En cinco de los casos, las gestantes expresaron sentimientos considerados positivos cuando hubo aceptación, soluciones o resolución de su situación. 
Las mujeres que vivieron la DGM, expresaron aceptación, adaptación y control al reconciliarse con su situación, aceptar la enfermedad y adaptarse a las rutinas y tratamiento impuesto. Lo anterior, fue posible gracias al desarrollo del sentido de la responsabilidad por cuidar de sí mismas para dar a su hijo un comienzo de vida saludable; y la superación de diferentes obstáculos lo cual mejoró la confianza en ellas mismas para enfrentar la enfermedad. Esto último también estuvo presente en las gestantes que convivían con el $\mathrm{VIH}$, ante la esperanza de reducción de la trasmisión del virus a sus hijos que significó el tratamiento retroviral.

En el caso de las mujeres que se enfrentaron a gestaciones multifetales, los sentimientos positivos que emergieron fueron la adaptación, posterior a la reducción embrionaria al sentir una situación de normalidad en su embarazo; y de aceptación de la imagen de sí misma como madre cuidadora de sus hijos.

\section{Tema 2. Experiencias de las mujeres frente a un embarazo de alto riesgo producto de la edad reproductiva temprana o tardía.}

\section{Experiencias negativas frente a la gestación}

Soporte deficiente en redes de apoyo primarias. En cuatro de los estudios, las adolescentes manifestaron que fueron rechazadas por sus padres y sus parejas; además de esto, $\mathrm{Hoga}^{25}$ registró en su artículo que se presentaba una relación de inferioridad de género, pues varias adolescentes quedaron embarazadas solo para satisfacer a la pareja.

Relación embarazo-estudio-mercado laboral. Tres trabajos refirieron que a causa del embarazo adolescente se presentó deserción escolar, lo que dificultó posteriormente el ingreso de las mujeres al mercado laboral. Además, interrumpir sus estudios significó acabar con algunos sueños en sus vidas.

\section{Experiencias positivas frente a la gestación}

Redes de apoyo primarias. En un estudio, la gestación durante la adolescencia se vivió como una experiencia positiva al posibilitar la formalización de la relación de las adolescentes con su pareja. En el caso del estudio sobre el embarazo después de los 35 años, las mujeres relataron que la participación de su compañero en todas las etapas del embarazo y el parto fue esencial para su sano desarrollo, y permitió el mantenimiento de las actividades cotidianas.

Posibilidad de madurar. Esta sub categoría habla acerca del caso de un estudio en que la gestación significó para las adolescentes asumir una nueva identidad como madres y el abandono del consumo de drogas, alcohol y tabaco. También fue la "solución" a los problemas dentro de sus familias y la posibilidad de mejores condiciones de vida personal y familiar.

Relación embarazo-estudio-mercado laboral. Moreira, Costa, Lopes, Freitas, Souza y Carvalho ${ }^{26}$ documentaron que algunas adolescentes participantes en su investigación regresaron a estudiar, con la creencia de que solo a través de una mejor educación pueden conseguir trabajo y mejores condiciones económicas.

Espiritualidad. En dos estudios aparece la creencia en Dios como soporte durante el proceso de la gestación adolescente. Así, se atribuyó la causa de la gestación como 
un "Deseo divino", y al evaluar la posibilidad del aborto, manifestaron que solo Dios tenía el poder de hacer vivir o morir al hijo que esperaban.

\section{Sentimientos negativos generados por la gestación}

En los cinco trabajos revisados, la gestación durante la adolescencia generó sentimientos que pueden considerarse negativos, como el miedo, la inseguridad, preocupación y la incertidumbre al tener que enfrentar a sus padres con la noticia del embarazo; el arrepentimiento por el deseo de abortar y la resignación frente a la nueva situación que suponía el hijo que se esperaba.

Por otro lado, en el estudio de Parada y Tonete ${ }^{27}$ sobre la experiencia de la gestación en mayores de 35 años, los sentimientos que emergieron fueron de conflicto cuando la gestación no era deseada o esperada en mujeres de escasos recursos económicos.

\section{CONCLUSIONES}

En relación al primer tema, a pesar de las diferencias en las causas de las gestaciones de alto riesgo por una condición patológica, el análisis de las categorías reportadas en los artículos científicos es muy similar entre ellas. Lo anterior solo a excepción de las gestantes contagiadas con $\mathrm{VIH}$, donde los sentimientos y experiencias dan cuenta de otro fenómeno diferente y de mayor complejidad. En este sentido, es necesaria la profundización en el abordaje este fenómeno, y más teniendo en cuenta que esta problemática se ha convertido en una de tipo crónico y la mujer en edad reproductiva, uno de sus blancos.

Sobre el segundo tema, el análisis no reporta aspectos novedosos en la investigación en relación al embarazo adolescente: una nueva opción de vida, la falta de redes de apoyo durante el evento y la deserción académica, entre otros. Por otro lado, un tema novedoso y poco explorado son las experiencias de las mujeres que viven una gestación después de los 35 años, o las conocidas "añosas". Este es un evento creciente en el mundo en desarrollo, dado los intereses y roles femeninos actuales.

En el quehacer diario, es preciso reforzar por parte de Enfermería el papel de las redes de apoyo para las mujeres que vivencian una gestación de alto riesgo como una forma de soporte esencial en estos momentos críticos, al resaltar el valor y papel que juegan estas redes primarias durante el padecimiento.

A pesar de las diferencias en los contextos de los estudios analizados, es inminente la revisión de los ambientes hospitalarios, y las condiciones de ocio y esparcimiento durante la estancia de las gestantes en su hospitalización; así como las posibilidades que ofrecen para la interacción con su familia. Asimismo, la revisión del manejo del lenguaje por parte de los profesionales, el cual fue referido como muy técnico para la comprensión de las mujeres.

Este estudio demostró que la investigación en este tema tiene mucho terreno por donde andar, reafirmando la deuda del quehacer investigativo para dar respuesta a una problemática a la que se ha respondido con éxito desde el saber biomédico, pero no desde el campo de la compresión de lo subjetivo del ser humano. En este sentido, la investigación cualitativa ha demostrado su utilidad en traer las voces de las mujeres que han padecido una gestación de alto riesgo; sin embargo, debe ampliarse su 
mirada y pensarse en las bondades que ofrece la triangulación de técnicas y participantes, que como plantea Minayo ${ }^{28}$, en lugar de restringirse a una fuente de datos permite multiplicar las tentativas de abordaje.

En esta misma lógica, es imprescindible reafirmar la inclusión de la perspectiva de las mujeres y sus familias que viven una gestación de alto riesgo, tanto en la formación de futuros enfermeros como en los planes de cuidado de enfermería.

Finalmente, se reafirma que una gestación de alto riesgo es un evento complejo y subjetivo; imposible de considerar solo como un suceso biológico. Implica cambios fisiológicos, psicológicos, sociales, económicos, culturales y espirituales que afectan directamente a la madre y su hijo. Desde esta perspectiva debe pensarse el cuidado de enfermería.

\section{BIBLIOGRAFÍA}

${ }^{1}$ Ministerio de la Protección social de Colombia-Organización Mundial de la SaludInstituto Nacional de Salud. Situación de salud de Colombia. Indicadores básicos. Bogotá, 2010. P:1-28.

${ }^{2}$ Carvalheira AP, Tonete VL, Parada MG. Sentimientos y percepciones de mujeres en el ciclo embarazo-puerperio que sobrevivieron a una morbosidad materna grave. Rev. Latino-Am. Enfermagem. 2010;18(6):1187-1194.

${ }^{3}$ Filippi V, Alihonou E, Mukantaganda S, Graham WJ, Ronsmans C. Near misses maternal morbidity and mortality. Lancet. 1998;351:145-156.

4 Schwarcz R; Duverges CA; Gonzalo Á; Fescina R. Obstetricia. Buenos Aires: El Ateneo, 1997.

${ }^{5}$ Salinas AM. Percepción de riesgo y actitud hacia la anticoncepción en mujeres cob embarazos de alto riesgo. Salud Pública Mex. 1994;36:513-520.

6 Cordero MZ, González GJ. factores socio económicos y de servicios de salud asociados con la mortalidad materna: una revisión. Rev.cienc.biomed. 2011;2(1):7785.

7 Aristizábal JP et al. Paciente obstétrica de alto riesgo: ¿Dónde debe realizarse su cuidado periparto? Revista Colombiana de Obstetricia y Ginecología 2005;56(2):166175.

${ }^{8}$ Manrique R, Rivero A, Ortunio M, Rivas M, Cardozo R, Guevara H. Parto pretérmino en adolescentes. Rev Obstet Ginecol Venez 2008;68(3):144-149.

${ }^{9}$ Husserl E. Experiencia y juicio. México: UNAM, 1980.

${ }^{10}$ Dilthey W. La esencia de la filosofía. Buenos Aires: Losada, 1944.

${ }^{11}$ Souza JP, Cecatti JG, Parpinelli MA, Krupa F, Osis M. An emerging "maternal nearmiss syndrome": narratives of women who almost died during pregnancy and childbirth. Birth. 2009;36(2):149-158.

${ }^{12}$ Vecino Al. Determinants of demand for antenatal care in Colombia. Health Policy. 2008;86:363-72.

${ }^{13}$ Hoyos C. Un modelo para investigación documental. Guía teórico-práctica sobre construcción de estados del arte con importantes reflexiones sobre la investigación. Medellín: Señal Editora, 2000.

${ }^{14}$ Bautista NP. Proceso de la investigación cualitativa: epistemología, metodología y aplicaciones. Bogotá: Manual Moderno, 2011.

${ }^{15}$ Duley L. The global impact of pre-eclampsia and eclampsia. Semin Perinatol 2009 33:130-137.

${ }^{16}$ Vigil P. Maternal deaths due to eclampsia and HELLP syndrome. Int $\mathrm{J}$ Gynecol Obstet. 2009;104:90-94. 
17 Jabeen M, Yakoob M, Imdad A, Bhutta Z. Impact of interventions to prevent and manage preeclampsia and eclampsia on stillbirths. BMC Public Health 2011;11(3):617.

${ }^{18}$ Huarte M, Modroño A, Larrañaga C. Conducta ante los estados hipertensivos del embarazo An. Sist. Sanit. Navar. 2009;32:91-103.

${ }^{19}$ Comisión Económica para América Latina y el Caribe (CEPAL). Informe anual 2011

El salto de la autonomía. De los márgenes al centro. Naciones Unidas: Santiago de Chile, 2011. P: 1-97.

${ }^{20}$ Souza N, Araújo AC, Azevedo G, Jerônimo SM, Barbosa L, Sousa NM. Percepção materna com o nascimento prematuro e vivência da gravidez com pré-eclampsia. Rev. Saúde Pública 2007;41(5):704-710.

21 Persson M, Winkvist A, Mogren I. 'From stun to gradual balance'-women's experiences of living with gestational diabetes mellitus. Scandinavian journal of caring sciences 2010;24(3):454-462.

${ }^{22}$ Araújo MA, Silveira C, Silveira C, Melo S. Vivências de gestantes e puérperas com o diagnóstico do HIV. Rev. bras. enferm. 2008;61(5):589-594.

${ }^{23}$ Ross R, Sawatphanit W, Draucker C, Suwansujarid T. The lived experiences of HIVpositive, pregnant women in Thailand. Health care for women international 2007; 28(8):731-744.

${ }^{24}$ Barlow J, Hainsworth J, Thornton S. Women's experiences of hospitalization with hypertension during pregnancy: feeling a fraud. Journal Of Reproductive \& Infant Psychology 2008;26(3):157-167.

${ }^{25}$ Hoga A. Adolescent maternity in a low income community: experiences revealed by oral history. Rev. Latino-Am. Enfermagem. 2008;16(2):280-286.

${ }^{26}$ Moreira RCR, Costa JRA, Lopes RLM, Freitas MYGS, Souza L, Carvalho MA. Gravidez na adolescência e vida escolar: experiências de alunas de uma escola pública. Rev enferm UFPE. 2010;4(2):524-32.

${ }^{27}$ Parada CM, Tonete VL. Experiência da gravidez após os 35 anos de mulheres com baixa renda. Esc. Anna Nery 2009;13(2):385-392.

${ }^{28}$ Minayo MC. El desafío del conocimiento. Investigación cualitativa en salud. Buenos Aires: Lugar Editorial, 1995. 
Anexo 1. Listado de artículos consultados.

\begin{tabular}{|c|c|c|c|c|}
\hline No. & Autores & Título & Año & Fuente \\
\hline 1 & $\begin{array}{l}\text { Rocha CR, } \\
\text { Quaresma MLJ. }\end{array}$ & $\begin{array}{l}\text { A percepção das gestantes } \\
\text { especiais: bases para o } \\
\text { cuidado de enfermagem. }\end{array}$ & 2009 & $\begin{array}{ll}\text { Revista } & \text { de } \\
\text { Pesquisa: } & \\
\text { Cuidado } & \text { é } \\
\text { fundamental } & \\
\text { (1)2;132-143. } & \end{array}$ \\
\hline 2 & $\begin{array}{lc}\text { Markovic } & \text { M, } \\
\text { Manderson } & \mathrm{L}, \\
\text { Schaper } & \mathrm{H}, \\
\text { Brennecke S. } & \\
\end{array}$ & $\begin{array}{l}\text { Maternal identity change as a } \\
\text { consequence of antenatal } \\
\text { hospitalization. }\end{array}$ & 2006 & $\begin{array}{l}\text { Health care for } \\
\text { women } \\
\text { international } \\
27(9): 762-776\end{array}$ \\
\hline 3 & $\begin{array}{ll}\text { Carvalheira } & \text { AP, } \\
\text { Tonete } & \text { VL, } \\
\text { Parada CM. } & \end{array}$ & $\begin{array}{l}\text { Sentimientos y percepciones } \\
\text { de mujeres en el } \\
\text { embarazo-puerperio } \\
\text { sobrevivieron que } \\
\text { morbosidad materna grave. }\end{array}$ & 2010 & $\begin{array}{l}\text { Rev. Latino-Am. } \\
\text { Enfermagem. } \\
\text { 18(6):1187-1194. }\end{array}$ \\
\hline 4 & $\begin{array}{l}\text { Vasconcelos D, } \\
\text { Pinheiro AC, Clara } \\
\text { ta Í, Medeiros A. }\end{array}$ & $\begin{array}{l}\text { Percepções e Sentimentos de } \\
\text { Gestantes e Puérperas sobre } \\
\text { a Pré-Eclâmpsia. }\end{array}$ & 2009 & $\begin{array}{l}\text { Rev. salud } \\
\text { pública } \\
11(3): 347-358\end{array}$ \\
\hline 5 & $\begin{array}{ll}\text { Barlow } & \mathrm{J}, \\
\text { Hainsworth } & \mathrm{J}, \\
\text { Thornton S. } & \end{array}$ & $\begin{array}{lr}\text { Women's experiences } & \text { of } \\
\text { hospitalization } & \text { with } \\
\text { hypertension } & \text { during } \\
\text { pregnancy: feeling a fraud. }\end{array}$ & 2008 & $\begin{array}{l}\text { Journal of } \\
\text { reproductive \& } \\
\text { infant psychology } \\
6(3): 157-167\end{array}$ \\
\hline 6 & $\begin{array}{l}\text { Souza N, Araújo } \\
\text { AC, Azevedo G, } \\
\text { Jerônimo SM, } \\
\text { Barbosa L, Sousa } \\
\text { NM. }\end{array}$ & $\begin{array}{l}\text { Percepção materna com o } \\
\text { nascimento prematuro é } \\
\text { vivência da gravidez com pré- } \\
\text { eclampsia. }\end{array}$ & 2007 & $\begin{array}{l}\text { Rev. Saúde } \\
\text { Pública } \\
\text { 41(5):704-710. }\end{array}$ \\
\hline 7 & $\begin{array}{ll}\text { Persson } & \text { M, } \\
\text { Winkvist } & \text { A, } \\
\text { Mogren I. } & \end{array}$ & $\begin{array}{l}\text { From stun to gradual balance'- } \\
\text { women's experiences of living } \\
\text { with gestational diabetes } \\
\text { mellitus. }\end{array}$ & 2010 & $\begin{array}{l}\text { Scandinavian } \\
\text { journal of caring } \\
\text { sciences } \\
24(3): 454-462 . \\
\end{array}$ \\
\hline 8 & Berg M. & $\begin{array}{l}\text { Pregnancy and Diabetes: How } \\
\text { women handle the challenges. }\end{array}$ & 2005 & $\begin{array}{l}\text { Journal } \\
\text { perinatal } \\
\text { education. } \\
14(3): 23-32 .\end{array}$ \\
\hline 9 & $\begin{array}{l}\text { Araújor MA, } \\
\text { Silveira C, Silveira } \\
\text { C, Melo S. }\end{array}$ & $\begin{array}{l}\text { Vivências de } \\
\text { puérperas com } \\
\text { do HIV. } \\
\text { do Hiagnóstico } \\
\end{array}$ & 2008 & $\begin{array}{l}\text { Rev. bras. } \\
\text { enferm. 61(5): } \\
\text { 589-594. }\end{array}$ \\
\hline 10 & $\begin{array}{lr}\text { Ross } & \text { R, } \\
\text { Sawatphanit } & \text { W, } \\
\text { Draucker } & \text { C, } \\
\text { Suwansujarid T. }\end{array}$ & $\begin{array}{l}\text { The lived experiences of HIV- } \\
\text { positive, pregnant women in } \\
\text { Thailand. }\end{array}$ & 2007 & $\begin{array}{l}\text { Health care for } \\
\text { women } \\
\text { international } \\
28(8): 731-744 \text {. }\end{array}$ \\
\hline 11 & $\begin{array}{l}\text { Costa M, Bezerra } \\
\text { JG, Andrade MG, } \\
\text { Veríssimo Ml, } \\
\text { Carvalho RM, De } \\
\text { Vasconcelos AR. }\end{array}$ & $\begin{array}{l}\text { Gestación de riesgo } \\
\text { percepción y sentimientos de } \\
\text { las mujeres embarazadas con } \\
\text { amniorrexe prematuro. }\end{array}$ & 2010 & $\begin{array}{l}\text { Enfermería global } \\
20: 1-12 \text {. }\end{array}$ \\
\hline 12 & $\begin{array}{l}\text { Amar J, Pesellín } \\
\text { M, Tirado D. }\end{array}$ & $\begin{array}{l}\text { VÍNCULO DE APEGO QUE } \\
\text { ESTABLECEN PADRES CON }\end{array}$ & 2009 & $\begin{array}{l}\text { Salud Uninorte } \\
\text { 25(2): 232-244. }\end{array}$ \\
\hline
\end{tabular}




\begin{tabular}{|c|c|c|c|c|}
\hline & & $\begin{array}{l}\text { HIJOS NACIDOS DE } \\
\text { EMBARAZOS MÚLTIPLES. }\end{array}$ & & \\
\hline 13 & $\begin{array}{l}\text { Wang HL, } \quad \text { Yu } \\
\text { Chao YM. }\end{array}$ & $\begin{array}{lcr}\text { Lived } & \text { experiences } & \text { of } \\
\text { taiwanese } & \text { women } & \text { with } \\
\text { multifetal pregnancies } & \text { who } \\
\text { receive fetal reduction. } & \end{array}$ & 2006 & $\begin{array}{l}\text { Journal of nursing } \\
\text { research } 14(2) \text { : } \\
143-154 .\end{array}$ \\
\hline 14 & $\begin{array}{ll}\text { Leichtentritt } & \mathrm{R}, \\
\text { Blumenthal } & \mathrm{N}, \\
\text { Elyassi } & \mathrm{A}, \\
\text { Rotmensch } & \mathrm{S} .\end{array}$ & $\begin{array}{l}\text { High-risk pregnancy and } \\
\text { hospitalization: the women's } \\
\text { voices. }\end{array}$ & 2005 & $\begin{array}{l}\text { Health } \quad \& \\
\text { social }{ }^{\text {work }} \\
30(1): 39-47 .\end{array}$ \\
\hline 15 & $\begin{array}{lr}\text { Moreirararar } & \text { RCR, } \\
\text { Costa JRA, Lopes } \\
\text { RLM, Freitas } \\
\text { MYGS, Souza L, } \\
\text { Carvalho MA. }\end{array}$ & $\begin{array}{l}\text { Gravidez na adolescência e } \\
\text { vida escolar: experiências de } \\
\text { alunas de uma escola pública. }\end{array}$ & 2010 & $\begin{array}{lr}\text { Rev } & \text { enferm } \\
\text { UFPE. } & 4(2): 524- \\
32 . & \end{array}$ \\
\hline 16 & $\begin{array}{l}\text { Soto VI, Durán } \\
\text { MM. }\end{array}$ & $\begin{array}{l}\text { Teenagers Want to be } \\
\text { Mothers... But.. }\end{array}$ & 2010 & $\begin{array}{l}\text { av. enferm 28:16- } \\
28 \text {. }\end{array}$ \\
\hline 17 & $\begin{array}{l}\text { Ortiz G, Bolaños } \\
\text { R, Rivera A, } \\
\text { Cerezo AM. }\end{array}$ & $\begin{array}{l}\text { Proceso de vivir un embarazo } \\
\text { en la adolescencia. }\end{array}$ & 2010 & $\begin{array}{l}\text { Desarrollo Científ } \\
\text { Enferm 18(1):30- } \\
33 .\end{array}$ \\
\hline 18 & $\begin{array}{l}\text { Prías HE, Miranda } \\
\text { C. }\end{array}$ & $\begin{array}{l}\text { Experiencias de adolescentes } \\
\text { embarazadas en control } \\
\text { prenatal. }\end{array}$ & 2009 & $\begin{array}{l}\text { Aquichan 9(1):93- } \\
105 .\end{array}$ \\
\hline 19 & Hoga A. & $\begin{array}{l}\text { Adolescent maternity in a low } \\
\text { income community: } \\
\text { experiences revealed by oral } \\
\text { history. }\end{array}$ & 2008 & $\begin{array}{l}\text { Rev. Latino-Am. } \\
\text { Enfermagem. } \\
\text { 16(2):280-286. }\end{array}$ \\
\hline 20 & $\begin{array}{l}\text { Parada } \\
\text { Tonete VL. }\end{array}$ & $\begin{array}{l}\text { Experiência da gravidez após } \\
\text { os } 35 \text { anos de mulheres com } \\
\text { baixa renda. }\end{array}$ & 2009 & $\begin{array}{l}\text { Esc. Anna Nery } \\
\text { 13(2):385-392. }\end{array}$ \\
\hline
\end{tabular}

Fuente: Los autores, 2012.

ISSN 1695-6141 